\title{
Necessary and sufficient conditions for the solvability of inverse problem for a class of Dirac operators
}

\author{
KH. R. Mamedov and O. Akcay
}




\title{
NECESSARY AND SUFFICIENT CONDITIONS FOR THE SOLVABILITY OF INVERSE PROBLEM FOR A CLASS OF DIRAC OPERATORS
}

\author{
KH. R. MAMEDOV AND O. AKCAY \\ Received 01 May, 2014
}

\begin{abstract}
In this paper we consider a problem for the first order Dirac differential equations system with spectral parameter dependent on boundary condition. The asymptotic behaviors of eigenvalues, eigenfunctions and normalizing numbers of this system are investigated. The expansion formula with respect to eigenfunctions is obtained and Parseval equality is given. The main theorem on necessary and sufficient conditions for the solvability of inverse problem is proved and the algorithm of reconstruction of potential from spectral data (the sets of eigenvalues and normalizing numbers) is given.
\end{abstract}

2010 Mathematics Subject Classification: 34A55; 34L40

Keywords: Dirac operator, eigenvalues and normalizing numbers, inverse problem, necessary and sufficient conditions

\section{INTRODUCTION}

We consider the boundary value problem generated by one dimensional Dirac differential equations system

$$
B y^{\prime}+\Omega(x) y=\lambda y, \quad 0<x<\pi
$$

with boundary condition

$$
\begin{gathered}
U(y):=y_{1}(0)=0, \\
V(y):=\left(\lambda+h_{1}\right) y_{1}(\pi)+h_{2} y_{2}(\pi)=0,
\end{gathered}
$$

where

$$
B=\left(\begin{array}{cc}
0 & 1 \\
-1 & 0
\end{array}\right), \quad \Omega(x)=\left(\begin{array}{cc}
p(x) & q(x) \\
q(x) & -p(x)
\end{array}\right), \quad y=\left(\begin{array}{c}
y_{1}(x) \\
y_{2}(x)
\end{array}\right),
$$

$p(x), q(x)$ are real valued functions in $L_{2}(0, \pi), \lambda$ is a spectral parameter, $h_{1}$ and $h_{2}$ are real numbers, $h_{2}>0$.

In the finite interval, the inverse problems for Dirac differential equations system by different spectral characteristics (e.g., one spectrum and norming constants or two spectra or spectral function) are solved in $[2,6,10,11,18,20,21]$. When spectral 
parameter contained in boundary condition, reconstruction of potential from spectral function is carried out in [18] and the uniqueness of the inverse problem for Dirac operator according to Weyl function is worked in [3]. Inverse spectral problem for Dirac operator with potentials belong entrywise to $L_{p}(0,1)$; for some $p \in[1, \infty)$, was studied in [2] and in this work, not only Gelfand-Levitan-Marchenko method but also Krein method [12] was used. Using Weyl-Titschmarsh function, direct and inverse problems for Dirac type-system were investigated in $[8,22]$. For weighted Dirac systems, inverse spectral problems was examined in [25].

On a positive half line, inverse scattering problem for a system of Dirac equations of order $2 n$ is completely solved in [9] and when boundary condition involving spectral parameter, for Dirac operator, inverse scattering problem worked in $[4,17]$. The applications of Dirac differential equations system has been widespread in various areas of physics, such as [5, 23, 24] ever since Dirac equation was discovered to be associated with nonlinear wave equation in [1].

This paper is organized as follows: In Section 2, the asymptotic formulas of eigenvalues, eigenfunctions and normalizing numbers of the boundary value problem (1.1), (1.2) are investigated. By the eigenfunctions, completeness theorem is proved and expansion formula is obtained. Parseval equality is given. In Section 3, the main equation namely Gelfand-Levitan-Marchenko type equation is derived. In Section 4 , we show that the boundary value problem $(1.1),(1.2)$ can be uniquely determined from its eigenvalues and normalizing numbers. Finally, in Section 5, the solution of the inverse problem is obtained. Let's express this more clearly. We can state the inverse problem for a system of Dirac equations in the following way: let $\lambda_{n}$ and $\alpha_{n},(n \in \mathbb{Z})$ are respectively eigenvalues and normalizing numbers of boundary value problem (1.1), (1.2) and the quantities $\left\{\lambda_{n}, \alpha_{n}\right\},(n \in \mathbb{Z})$ are called spectral data. Knowing the spectral data $\left\{\lambda_{n}, \alpha_{n}\right\},(n \in \mathbb{Z})$ to indicate a method of determining the potential $\Omega(x)$ and to find necessary and sufficient conditions for $\lambda_{n}$ and $\alpha_{n},(n \in \mathbb{Z})$ to be the spectral data of a problem (1.1),(1.2), for this, we derive differential equation, Parseval equality and boundary conditions. The main theorem on the necessary and sufficient conditions for the solvability of inverse problem is proved and then algorithm of the construction of the function $\Omega(x)$ by spectral data is given. Note that throughout this paper, we use the following notation: $\tilde{\phi}$ denotes the transposed matrix of $\phi$.

\section{PRELIMINARIES}

The inner product in Hilbert space $H=L_{2}\left(0, \pi ; \mathbb{C}^{2}\right) \oplus \mathbb{C}$ is defined by

$$
\langle Y, Z\rangle:=\int_{0}^{\pi}\left[y_{1}(x) \bar{z}_{1}(x)+y_{2}(x) \bar{z}_{2}(x)\right] d x+\frac{1}{h_{2}} y_{3} \bar{z}_{3},
$$


where

$$
Y=\left(\begin{array}{c}
y_{1}(x) \\
y_{2}(x) \\
y_{3}
\end{array}\right) \in H, \quad Z=\left(\begin{array}{c}
z_{1}(x) \\
z_{2}(x) \\
z_{3}
\end{array}\right) \in H
$$

Let us define

$$
L(Y):=\left(\begin{array}{c}
l(y) \\
-h_{1} y_{1}(\pi)-h_{2} y_{2}(\pi)
\end{array}\right)
$$

with

$$
D(L)=\left\{\begin{array}{c}
Y \mid Y=\left(y_{1}(x), y_{2}(x), y_{3}\right)^{T} \in H, y_{1}(x), y_{2}(x) \in A C[0, \pi], \\
y_{3}=y_{1}(\pi), y_{1}(0)=0, l(y) \in L_{2}\left(0, \pi ; \mathbb{C}^{2}\right),
\end{array}\right\}
$$

where

$$
l(y)=\left(\begin{array}{c}
y_{2}^{\prime}+p(x) y_{1}+q(x) y_{2} \\
-y_{1}^{\prime}+q(x) y_{1}-p(x) y_{2}
\end{array}\right) .
$$

The boundary value problem (1.1), (1.2) is equivalent to the equation $L Y=\lambda Y$.

Let $\varphi(x, \lambda)$ and $\psi(x, \lambda)$ be solutions of the system (1.1) satisfying the initial conditions

$$
\varphi(0, \lambda)=\left(\begin{array}{c}
0 \\
-1
\end{array}\right), \quad \psi(\pi, \lambda)=\left(\begin{array}{c}
h_{2} \\
-\lambda-h_{1}
\end{array}\right) .
$$

The solution $\varphi(x, \lambda)$ has the following representation (see [3])

$$
\varphi(x, \lambda)=\varphi_{0}(x, \lambda)+\int_{0}^{x} K(x, t) \varphi_{0}(t, \lambda) d t,
$$

where $\varphi_{0}(x, \lambda)=\left(\begin{array}{c}\sin \lambda x \\ -\cos \lambda x\end{array}\right), K_{i j}(x,.) \in L_{2}(0, \pi), i, j=1,2$ for fixed $x \in[0, \pi]$ and $K(x, t)$ is the solution of the problem

$$
\begin{aligned}
B K_{x}^{\prime}(x, t)+K_{t}^{\prime}(x, t) B & =-\Omega(x) K(x, t), \\
\Omega(x) & =K(x, x) B-B K(x, x), \\
K_{11}(x, 0) & =K_{21}(x, 0)=0 .
\end{aligned}
$$

Formula (2.3) gives the relation between the kernel $K(x, t)$ and the coefficient of $\Omega(x)$ of equation (1.1).

The characteristic function $\Delta(\lambda)$ of the problem $L$ is

$$
\Delta(\lambda):=W[\varphi(x, \lambda), \psi(x, \lambda)]=\varphi_{2}(x, \lambda) \psi_{1}(x, \lambda)-\varphi_{1}(x, \lambda) \psi_{2}(x, \lambda),
$$

where $W[\varphi(x, \lambda), \psi(x, \lambda)]$ is the Wronskian of the solutions $\varphi(x, \lambda)$ and $\psi(x, \lambda)$ and independent of $x \in[0, \pi]$. The zeros of $\Delta(\lambda)$ coincide with the eigenvalues $\lambda_{n}$ of problem $L$. The functions $\varphi(x, \lambda)$ and $\psi(x, \lambda)$ are eigenfunctions and there exists a sequence $\beta_{n}$ such that

$$
\psi\left(x, \lambda_{n}\right)=\beta_{n} \varphi\left(x, \lambda_{n}\right), \quad \beta_{n} \neq 0 .
$$


Normalizing numbers are

$$
\alpha_{n}:=\int_{0}^{\pi}\left(\left|\varphi_{1}\left(x, \lambda_{n}\right)\right|^{2}+\left|\varphi_{2}\left(x, \lambda_{n}\right)\right|^{2}\right) d x+\frac{1}{h_{2}}\left|\varphi_{1}\left(\pi, \lambda_{n}\right)\right|^{2} .
$$

The following relation holds (see [16]):

$$
\dot{\Delta}\left(\lambda_{n}\right)=\beta_{n} \alpha_{n},
$$

where $\dot{\Delta}(\lambda)=\frac{d}{d \lambda} \Delta(\lambda)$.

Theorem 1. i) The eigenvalues $\lambda_{n},(n \in \mathbb{Z})$ of boundary value problem (1.1), (1.2) are

$$
\lambda_{n}=\lambda_{n}^{0}+\epsilon_{n}, \quad\left\{\epsilon_{n}\right\} \in l_{2},
$$

where $\lambda_{n}^{0}=n$ are zeros of function $\lambda \sin \lambda \pi$. For large $n$, the eigenvalues are simple.

ii) The eigenfunctions of the boundary value problem can be represented in the form

$$
\varphi\left(x, \lambda_{n}\right)=\left(\begin{array}{c}
\sin n x \\
-\cos n x
\end{array}\right)+\left(\begin{array}{c}
\zeta_{n}^{(1)}(x) \\
\zeta_{n}^{(2)}(x)
\end{array}\right),
$$

where $\sum_{n=-\infty}^{\infty}\left\{\left|\zeta_{n}^{(1)}(x)\right|^{2}+\left|\zeta_{n}^{(2)}(x)\right|^{2}\right\} \leq C$, here $C$ is a positive number.

iii) Normalizing numbers of the problem (1.1), (1.2) are as follows

$$
\alpha_{n}=\pi+\tau_{n}, \quad\left\{\tau_{n}\right\} \in l_{2} .
$$

Proof. The proof of this theorem is given in [15].

We note that using (2.2), as $|\lambda| \rightarrow \infty$ uniformly in $x \in[0, \pi]$ the following asymptotic formulas are obtain:

$$
\begin{aligned}
& \varphi_{1}(x, \lambda)=\sin \lambda x+O\left(\frac{1}{|\lambda|} e^{|\operatorname{Im} \lambda| x}\right), \\
& \varphi_{2}(x, \lambda)=-\cos \lambda x+O\left(\frac{1}{|\lambda|} e^{|\operatorname{Im} \lambda| x}\right) .
\end{aligned}
$$

Substituting the asymptotic formulas (2.10) into

$$
\Delta(\lambda)=\left(\lambda+h_{1}\right) \varphi_{1}(\pi, \lambda)+h_{2} \varphi_{2}(\pi, \lambda),
$$

we get as $|\lambda| \rightarrow \infty$

$$
\Delta(\lambda)=\lambda \sin \lambda \pi+O\left(e^{|\operatorname{Im} \lambda| \pi}\right) .
$$

Proposition 1. The specification of the eigenvalues $\lambda_{n}(n \in \mathbb{Z})$ uniquely determines the characteristic function $\Delta(\lambda)$ by the formula

$$
\Delta(\lambda)=-\pi\left(\lambda_{0}^{2}-\lambda^{2}\right) \prod_{n=1}^{\infty} \frac{\left(\lambda_{n}^{2}-\lambda^{2}\right)}{n^{2}} .
$$


Proof. Since the function $\Delta(\lambda)$ is entire function of order 1, from Hadamard's theorem (see [13]) using (2.11) we obtain (2.12).

Theorem 2. a) The system of eigenfunctions $\left\{\varphi\left(x, \lambda_{n}\right)\right\},(n \in \mathbb{Z})$ of boundary value problem (1.1), (1.2) is complete in space $L_{2}\left(0, \pi ; \mathbb{C}^{2}\right)$.

b) Let $f(x), x \in[0, \pi]$ be an absolutely continuous function. Then the expansion formula

$$
\begin{aligned}
f(x) & =\sum_{n=-\infty}^{\infty} a_{n} \varphi\left(x, \lambda_{n}\right), \\
a_{n} & =\frac{1}{\alpha_{n}}\left\langle f(x), \varphi\left(x, \lambda_{n}\right)\right\rangle
\end{aligned}
$$

is valid and the series converges uniformly with respect to $x \in[0, \pi]$. For $f(x) \in$ $L_{2}\left(0, \pi ; \mathbb{C}^{2}\right)$ series $(2.13)$ converges in $L_{2}\left(0, \pi ; \mathbb{C}^{2}\right)$, moreover, Parseval equality holds

$$
\|f\|^{2}=\sum_{n=-\infty}^{\infty} \alpha_{n}\left|a_{n}\right|^{2} .
$$

Proof. The proof of this theorem is obtained in [15].

\section{MAIN EQUATION}

Theorem 3. For each fixed $x \in(0, \pi]$ the kernel $K(x, t)$ satisfies the following equation:

$$
F(x, t)+K(x, t)+\int_{0}^{x} K(x, \xi) F(\xi, t) d \xi=0, \quad 0<t<x,
$$

where

$$
F(x, t)=\sum_{n=-\infty}^{\infty}\left[\frac{1}{\alpha_{n}} \varphi_{0}\left(x, \lambda_{n}\right) \tilde{\varphi}_{0}\left(t, \lambda_{n}\right)-\frac{1}{\pi} \varphi_{0}\left(x, \lambda_{n}^{0}\right) \tilde{\varphi}_{0}\left(t, \lambda_{n}^{0}\right)\right] .
$$

Proof. Using the transformation operators (see [14]), according to (2.2) we have

$$
\varphi_{0}(x, \lambda)=\varphi(x, \lambda)+\int_{0}^{x} H(x, t) \varphi(t, \lambda) d t .
$$

It follows from (2.2) and (3.3) that

$$
\begin{aligned}
& \sum_{n=-N}^{N} \frac{1}{\alpha_{n}} \varphi\left(x, \lambda_{n}\right) \tilde{\varphi}_{0}\left(t, \lambda_{n}\right) \\
& \quad=\sum_{n=-N}^{N} \frac{1}{\alpha_{n}} \varphi_{0}\left(x, \lambda_{n}\right) \tilde{\varphi}_{0}\left(t, \lambda_{n}\right)+\int_{0}^{x} K(x, \xi) \sum_{n=-N}^{N} \frac{1}{\alpha_{n}} \varphi_{0}\left(\xi, \lambda_{n}\right) \tilde{\varphi}_{0}\left(t, \lambda_{n}\right) d \xi
\end{aligned}
$$


and

$$
\begin{aligned}
& \sum_{n=-N}^{N} \frac{1}{\alpha_{n}} \varphi\left(x, \lambda_{n}\right) \tilde{\varphi}_{0}\left(t, \lambda_{n}\right) \\
& \quad=\sum_{n=-N}^{N} \frac{1}{\alpha_{n}} \varphi\left(x, \lambda_{n}\right) \tilde{\varphi}\left(t, \lambda_{n}\right)+\sum_{n=-N}^{N} \frac{1}{\alpha_{n}} \varphi\left(x, \lambda_{n}\right) \int_{0}^{t} \tilde{\varphi}\left(\xi, \lambda_{n}\right) \tilde{H}(t, \xi) d \xi .
\end{aligned}
$$

Using the last two equalities, we obtain

$$
\begin{aligned}
& \sum_{n=-N}^{N}\left[\frac{1}{\alpha_{n}} \varphi\left(x, \lambda_{n}\right) \tilde{\varphi}\left(t, \lambda_{n}\right)-\frac{1}{\pi} \varphi_{0}\left(x, \lambda_{n}^{0}\right) \tilde{\varphi}_{0}\left(t, \lambda_{n}^{0}\right)\right] \\
= & \sum_{n=-N}^{N}\left[\frac{1}{\alpha_{n}} \varphi_{0}\left(x, \lambda_{n}\right) \tilde{\varphi}_{0}\left(t, \lambda_{n}\right)-\frac{1}{\pi} \varphi_{0}\left(x, \lambda_{n}^{0}\right) \tilde{\varphi}_{0}\left(t, \lambda_{n}^{0}\right)\right] \\
& +\int_{0}^{x} K(x, \xi) \sum_{n=-N}^{N}\left[\frac{1}{\alpha_{n}} \varphi_{0}\left(\xi, \lambda_{n}\right) \tilde{\varphi}_{0}\left(t, \lambda_{n}\right)-\frac{1}{\pi} \varphi_{0}\left(\xi, \lambda_{n}^{0}\right) \tilde{\varphi}_{0}\left(t, \lambda_{n}^{0}\right)\right] d \xi \\
& +\int_{0}^{x} K(x, \xi) \sum_{n=-N}^{N} \frac{1}{\pi} \varphi_{0}\left(\xi, \lambda_{n}^{0}\right) \tilde{\varphi}_{0}\left(t, \lambda_{n}^{0}\right) d \xi \\
& -\sum_{n=-N}^{N} \frac{1}{\alpha_{n}} \varphi\left(x, \lambda_{n}\right) \int_{0}^{t} \tilde{\varphi}\left(\xi, \lambda_{n}\right) \tilde{H}(t, \xi) d \xi
\end{aligned}
$$

or

$$
\Phi_{N}(x, t)=I_{N 1}(x, t)+I_{N 2}(x, t)+I_{N 3}(x, t)+I_{N 4}(x, t),
$$

where

$$
\begin{aligned}
& \Phi_{N}(x, t):=\sum_{n=-N}^{N}\left[\frac{1}{\alpha_{n}} \varphi\left(x, \lambda_{n}\right) \tilde{\varphi}\left(t, \lambda_{n}\right)-\frac{1}{\pi} \varphi_{0}\left(x, \lambda_{n}^{0}\right) \tilde{\varphi}_{0}\left(t, \lambda_{n}^{0}\right)\right], \\
& I_{N 1}(x, t): \sum_{n=-N}^{N}\left[\frac{1}{\alpha_{n}} \varphi_{0}\left(x, \lambda_{n}\right) \tilde{\varphi}_{0}\left(t, \lambda_{n}\right)-\frac{1}{\pi} \varphi_{0}\left(x, \lambda_{n}^{0}\right) \tilde{\varphi}_{0}\left(t, \lambda_{n}^{0}\right)\right], \\
& I_{N 2}(x, t):=\int_{0}^{x} K(x, \xi) \sum_{n=-N}^{N}\left[\frac{1}{\alpha_{n}} \varphi_{0}\left(\xi, \lambda_{n}\right) \tilde{\varphi}_{0}\left(t, \lambda_{n}\right)-\frac{1}{\pi} \varphi_{0}\left(\xi, \lambda_{n}^{0}\right) \tilde{\varphi}_{0}\left(t, \lambda_{n}^{0}\right)\right] d \xi, \\
& I_{N 3}(x, t):=\int_{0}^{x} K(x, \xi) \sum_{n=-N}^{N} \frac{1}{\pi} \varphi_{0}\left(\xi, \lambda_{n}^{0}\right) \tilde{\varphi}_{0}\left(t, \lambda_{n}^{0}\right) d \xi,
\end{aligned}
$$




$$
I_{N 4}(x, t):=\sum_{n=-N}^{N} \frac{1}{\alpha_{n}} \varphi\left(x, \lambda_{n}\right) \int_{0}^{t} \tilde{\varphi}\left(\xi, \lambda_{n}\right) \tilde{H}(t, \xi) d \xi .
$$

Let $f(x)$ be an absolutely continuous function. Then using expansion formula (2.13),

$$
\begin{aligned}
& \lim _{N \rightarrow \infty} \int_{0}^{\pi} \Phi_{N}(x, t) f(t) d t=\lim _{N \rightarrow \infty} \int_{0}^{\pi} \sum_{n=-N}^{N} \frac{1}{\alpha_{n}} \varphi\left(x, \lambda_{n}\right) \tilde{\varphi}\left(t, \lambda_{n}\right) f(t) d t \\
& -\lim _{N \rightarrow \infty} \int_{0}^{\pi} \sum_{n=-N}^{N} \frac{1}{\pi} \varphi_{0}\left(x, \lambda_{n}^{0}\right) \tilde{\varphi}_{0}\left(t, \lambda_{n}^{0}\right) f(t) d t=0 \\
& \lim _{N \rightarrow \infty} \int_{0}^{\pi} I_{N 1}(x, t) f(t) d t \\
& =\lim _{N \rightarrow \infty} \int_{0}^{\pi} \sum_{n=-N}^{N}\left[\frac{1}{\alpha_{n}} \varphi_{0}\left(x, \lambda_{n}\right) \tilde{\varphi}_{0}\left(t, \lambda_{n}\right)-\frac{1}{\pi} \varphi_{0}\left(x, \lambda_{n}^{0}\right) \tilde{\varphi}_{0}\left(t, \lambda_{n}^{0}\right)\right] f(t) d t \\
& =\int_{0}^{\pi} F(x, t) f(t) d t
\end{aligned}
$$

$$
\begin{aligned}
& \lim _{N \rightarrow \infty} \int_{0}^{\pi} I_{N 2}(x, t) f(t) d t=\lim _{N \rightarrow \infty} \int_{0}^{\pi} \int_{0}^{x} K(x, \xi) \sum_{n=-N}^{N}\left[\frac{1}{\alpha_{n}} \varphi_{0}\left(\xi, \lambda_{n}\right) \tilde{\varphi}_{0}\left(t, \lambda_{n}\right)\right. \\
& \left.-\frac{1}{\pi} \varphi_{0}\left(\xi, \lambda_{n}^{0}\right) \tilde{\varphi}_{0}\left(t, \lambda_{n}^{0}\right)\right] f(t) d \xi d t=\int_{0}^{\pi}\left(\int_{0}^{x} K(x, \xi) F(\xi, t) d \xi\right) f(t) d t, \quad(3.7) \\
& \lim _{N \rightarrow \infty} \int_{0}^{\pi} I_{N 3}(x, t) f(t) d t \\
& =\lim _{N \rightarrow \infty} \int_{0}^{\pi} \int_{0}^{x} K(x, \xi) \sum_{n=-N}^{N} \frac{1}{\pi} \varphi_{0}\left(\xi, \lambda_{n}^{0}\right) \tilde{\varphi}_{0}\left(t, \lambda_{n}^{0}\right) f(t) d \xi d t \\
& \quad=\int_{0}^{x} K(x, \xi) f(\xi) d \xi, \\
& \lim _{N \rightarrow \infty} \int_{0}^{\pi} I_{N 4}(x, t) f(t) d t \\
& =\lim _{N \rightarrow \infty} \int_{0}^{\pi}\left(\sum_{n=-N}^{N} \frac{1}{\alpha_{n}} \varphi\left(x, \lambda_{n}\right) \int_{0}^{t} \tilde{\varphi}\left(\xi, \lambda_{n}\right) \tilde{H}(t, \xi) d \xi\right) f(t) d t
\end{aligned}
$$




$$
=\int_{x}^{\pi} \tilde{H}(t, x) f(t) d t .
$$

We put $K(x, t)=H(x, t)=0$ for $x<t$. Using (3.4), (3.5), (3.6), (3.7), (3.8) and (3.9) we obtain

$$
\begin{aligned}
\int_{0}^{\pi} F(x, t) f(t) d t+\int_{0}^{\pi}\left(\int_{0}^{x} K(x, \xi) F(\xi, t) d \xi\right) f(t) d t \\
\\
\quad+\int_{0}^{x} K(x, \xi) f(\xi) d \xi-\int_{x}^{\pi} \tilde{H}(t, x) f(t) d t=0 .
\end{aligned}
$$

Since $f(x)$ can be chosen arbitrarily,

$$
F(x, t)+K(x, t)+\int_{0}^{x} K(x, \xi) F(\xi, t) d \xi-\tilde{H}(t, x)=0 .
$$

When $t<x$, this equality implies (3.1).

\section{THEOREM FOR THE SOLUTION OF THE INVERSE PROBLEM}

Lemma 1. For each fixed $x \in(0, \pi]$ the main equation (3.1) has a unique solution $K(x, t) \in L_{2}(0, x)$.

Proof. It suffices to prove that homogeneous equation

$$
g(t)+\int_{0}^{x} g(s) F(s, t) d s=0
$$

has only trivial solution $g(t)=0$. Let $g(t)=\left(g_{1}(t), g_{2}(t)\right) \in L_{2}(0, x)$ be a solution of the above equation and $g(t)=0$ for $t \in(x, \pi)$. Then

$$
(g(t), g(t))+\left(\int_{0}^{x} g(s) F(s, t) d s, g(t)\right)=0 .
$$

Using the expression (3.2), we get

$$
\begin{aligned}
\int_{0}^{x} & \left(g_{1}^{2}(t)+g_{2}^{2}(t)\right) d t \\
& +\sum_{n=-\infty}^{\infty} \frac{1}{\alpha_{n}} \int_{0}^{x}\left[\int_{0}^{x}\left(g_{1}(s) \sin \lambda_{n} s-g_{2}(s) \cos \lambda_{n} s\right) d s\right] g_{1}(t) \sin \lambda_{n} t d t \\
& -\sum_{n=-\infty}^{\infty} \frac{1}{\alpha_{n}} \int_{0}^{x}\left[\int_{0}^{x}\left(g_{1}(s) \sin \lambda_{n} s-g_{2}(s) \cos \lambda_{n} s\right) d s\right] g_{2}(t) \cos \lambda_{n} t d t \\
& -\sum_{n=-\infty}^{\infty} \frac{1}{\pi} \int_{0}^{x}\left[\int_{0}^{x}\left(g_{1}(s) \sin \lambda_{n}^{0} s-g_{2}(s) \cos \lambda_{n}^{0} s\right) d s\right] g_{1}(t) \sin \lambda_{n}^{0} t d t \\
& +\sum_{n=-\infty}^{\infty} \frac{1}{\pi} \int_{0}^{x}\left[\int_{0}^{x}\left(g_{1}(s) \sin \lambda_{n}^{0} s-g_{2}(s) \cos \lambda_{n}^{0} s\right) d s\right] g_{2}(t) \cos \lambda_{n}^{0} t d t
\end{aligned}
$$




$$
\begin{aligned}
= & \int_{0}^{x}\left(g_{1}^{2}(t)+g_{2}^{2}(t)\right) d t+\sum_{n=-\infty}^{\infty} \frac{1}{\alpha_{n}}\left(\int_{0}^{x}\left[g_{1}(t) \sin \lambda_{n} t-g_{2}(t) \cos \lambda_{n} t\right] d t\right)^{2} \\
& -\sum_{n=-\infty}^{\infty} \frac{1}{\pi}\left(\int_{0}^{x}\left[g_{1}(t) \sin \lambda_{n}^{0} t-g_{2}(t) \cos \lambda_{n}^{0} t\right] d t\right)^{2}=0 .
\end{aligned}
$$

Thus, it follows from the last relation that

$$
\begin{aligned}
\int_{0}^{x}\left(g_{1}^{2}(t)+g_{2}^{2}(t)\right) d t+\sum_{n=-\infty}^{\infty} \frac{1}{\alpha_{n}} & \left(\int_{0}^{x} g(t) \varphi_{0}\left(t, \lambda_{n}\right) d t\right)^{2} \\
& -\sum_{n=-\infty}^{\infty} \frac{1}{\pi}\left(\int_{0}^{x} g(t) \varphi_{0}\left(t, \lambda_{n}^{0}\right) d t\right)^{2}=0 .
\end{aligned}
$$

Using Parseval equality,

$$
\|g\|^{2}=\sum_{n=-\infty}^{\infty} \frac{1}{\pi}\left(\int_{0}^{x} g(t) \varphi_{0}\left(t, \lambda_{n}^{0}\right) d t\right)^{2}
$$

we have

$$
\sum_{n=-\infty}^{\infty} \frac{1}{\alpha_{n}}\left(\int_{0}^{x} g(t) \varphi_{0}\left(t, \lambda_{n}\right) d t\right)^{2}=0 .
$$

Since $\alpha_{n}>0$ and from statement a) in Theorem 2, the system $\left\{\varphi_{0}\left(t, \lambda_{n}\right)\right\},(n \in \mathbb{Z})$ is complete in $L_{2}\left(0, \pi ; \mathbb{C}^{2}\right)$, we obtain $g(t)=0$.

Theorem 4. Let $L\left(\Omega(x), h_{1}, h_{2}\right)$ and $\hat{L}\left(\hat{\Omega}(x), \hat{h}_{1}, \hat{h}_{2}\right)$ be two boundary value problems and

$$
\lambda_{n}=\hat{\lambda}_{n}, \quad \alpha_{n}=\hat{\alpha}_{n}, \quad(n \in \mathbb{Z}) .
$$

Then

$$
\Omega(x)=\hat{\Omega}(x) \text { a.e. on }(0, \pi), h_{1}=\hat{h}_{1}, h_{2}=\hat{h}_{2} .
$$

Proof. According to (3.2), $F(x, t)=\hat{F}(x, t)$. Then, from the main equation (3.1), we have $K(x, t)=\hat{K}(x, t)$. It follows from $(2.3)$ that $\Omega(x)=\hat{\Omega}(x)$ a.e. on $(0, \pi)$. Taking into account (2.2), we find $\varphi\left(x, \lambda_{n}\right)=\hat{\varphi}\left(x, \lambda_{n}\right)$. In consideration of (2.11), we get $\dot{\Delta}\left(\lambda_{n}\right) \equiv \dot{\hat{\Delta}}\left(\lambda_{n}\right)$ and from (2.6), $\beta_{n}=\hat{\beta}_{n}$. Thus, using (2.1) and (2.5), we obtain $h_{1}=\hat{h}_{1}, h_{2}=\hat{h}_{2}$.

\section{SOLUTION OF THE INVERSE PROBLEM}

Let the real numbers $\left\{\lambda_{n}, \alpha_{n}\right\},(n \in \mathbb{Z})$ of the form (2.7) and (2.9) be given. Using these numbers, we construct the function $F(x, t)$ by the formulas (3.2) and determine $K(x, t)$ from the main equation (3.1). 
Now, let us construct the functions $\varphi(x, \lambda)$ by the formula $(2.2), \Omega(x)$ by the formula (2.3), $\Delta(\lambda)$ by the formula (2.12) and $\beta_{n}$ by the formula (2.6) respectively, i.e.,

$$
\begin{aligned}
\varphi(x, \lambda) & :=\varphi_{0}(x, \lambda)+\int_{0}^{x} K(x, t) \varphi(t, \lambda) d t, \\
\Omega(x) & :=K(x, x) B-B K(x, x), \\
\Delta(\lambda) & :=-\pi\left(\lambda_{0}^{2}-\lambda^{2}\right) \prod_{n=1}^{\infty} \frac{\left(\lambda_{n}^{2}-\lambda^{2}\right)}{n^{2}}, \\
\beta_{n} & :=\frac{\dot{\Delta}\left(\lambda_{n}\right)}{\alpha_{n}} \neq 0 .
\end{aligned}
$$

The function $F(x, t)$ can be rewritten as follows:

$$
F(x, t)=\frac{1}{2}[a(x-t)+a(x+t) T],
$$

where

$$
a(x)=\sum_{n=-\infty}^{\infty}\left[\frac{1}{\alpha_{n}}\left(\begin{array}{cc}
\cos \lambda_{n} x & -\sin \lambda_{n} x \\
\sin \lambda_{n} x & \cos \lambda_{n} x
\end{array}\right)-\frac{1}{\pi}\left(\begin{array}{cc}
\cos n x & -\sin n x \\
\sin n x & \cos n x
\end{array}\right)\right]
$$

and $T=\left(\begin{array}{cc}-1 & 0 \\ 0 & 1\end{array}\right)$. It is shown analogously in ([7], Lemma 1.5.4) that the function $a(x) \in W_{2}^{1}[0,2 \pi]$.

\subsection{Derivation of the differential equation}

Theorem 5. The following relations hold:

$$
\begin{gathered}
B \varphi^{\prime}(x, \lambda)+\Omega(x) \varphi(x, \lambda)=\lambda \varphi(x, \lambda), \\
\varphi_{1}(0, \lambda)=0, \quad \varphi_{2}(0, \lambda)=-1 .
\end{gathered}
$$

Proof. Differentiating on $x$ and $t$ the main equation (3.1) respectively, we get

$$
\begin{gathered}
F_{x}^{\prime}(x, t)+K_{x}^{\prime}(x, t)+K(x, x) F(x, t)+\int_{0}^{x} K_{x}^{\prime}(x, \xi) F(\xi, t) d \xi=0, \\
F_{t}^{\prime}(x, t)+K_{t}^{\prime}(x, t)+\int_{0}^{x} K(x, \xi) F_{t}^{\prime}(\xi, t) d \xi=0 .
\end{gathered}
$$

It follows from (3.2) that

$$
F_{t}^{\prime}(x, t) B+B F_{x}^{\prime}(x, t)=0 .
$$

Since $F(x, 0) B S=0$, where $S=\left(\begin{array}{c}0 \\ -1\end{array}\right)$, using the main equation (3.1), we obtain

$$
K(x, 0) B S=0 \text {, }
$$


or

$$
K_{11}(x, 0)=K_{21}(x, 0)=0 .
$$

Multiplying the equation (5.3) on the left by $B$, we get

$$
B F_{x}^{\prime}(x, t)+B K_{x}^{\prime}(x, t)+B K(x, x) F(x, t)+\int_{0}^{x} B K_{x}^{\prime}(x, \xi) F(\xi, t) d \xi=0,
$$

and multiplying the equation (5.4) on the right by $B$, we find

$$
F_{t}^{\prime}(x, t) B+K_{t}^{\prime}(x, t) B+\int_{0}^{x} K(x, \xi) F_{t}^{\prime}(\xi, t) B d \xi=0 .
$$

Adding the last two equalities and using (5.5)

$$
\begin{aligned}
B K_{x}^{\prime}(x, t)+B K(x, x) & F(x, t)+\int_{0}^{x} B K_{x}^{\prime}(x, \xi) F(\xi, t) d \xi \\
& =-K_{t}^{\prime}(x, t) B+\int_{0}^{x} K(x, \xi) B F_{\xi}^{\prime}(\xi, t) d \xi \equiv I(x, t) .
\end{aligned}
$$

Integrating by parts in (5.7) and from (5.6),

$$
I(x, t)=-K_{t}^{\prime}(x, t) B+K(x, x) B F(x, t)-\int_{0}^{x} K_{\xi}^{\prime}(x, \xi) B F(\xi, t) d \xi
$$

is obtained. Substituting (5.8) into (5.7), we have

$$
\begin{aligned}
B K_{x}^{\prime}(x, t)+B K(x, x) F(x, t) & +K_{t}^{\prime}(x, t) B-K(x, x) B F(x, t) \\
& +\int_{0}^{x}\left[B K_{x}^{\prime}(x, \xi)+K_{\xi}^{\prime}(x, \xi) B\right] F(\xi, t) d \xi=0 .
\end{aligned}
$$

Multiplying (3.1) on the left by $\Omega(x)$ in form of (2.3) and adding to (5.9),

$$
\begin{aligned}
B K_{x}^{\prime}(x, t) & +K_{t}^{\prime}(x, t) B+\Omega(x) K(x, t) \\
& +\int_{0}^{x}\left[B K_{x}^{\prime}(x, \xi)+K_{\xi}^{\prime}(x, \xi) B+\Omega(x) K(x, \xi)\right] F(\xi, t) d \xi=0
\end{aligned}
$$

is obtained. Setting

$$
J(x, t):=B K_{x}^{\prime}(x, t)+K_{t}^{\prime}(x, t) B+\Omega(x) K(x, t),
$$

we can rewrite equation (5.10) as follows

$$
J(x, t)+\int_{0}^{x} J(x, \xi) F(\xi, t) d \xi=0 .
$$

According to Lemma 1, homogeneous equation (5.11) has only trivial solution, i.e.,

$$
B K_{x}^{\prime}(x, t)+K_{t}^{\prime}(x, t) B+\Omega(x) K(x, t)=0,0<t<x .
$$

Now, differentiating (2.2) and multiplying on the left by $B$, we have

$$
B \varphi_{x}^{\prime}(x, \lambda)=\lambda B\left(\begin{array}{c}
\cos \lambda x \\
\sin \lambda x
\end{array}\right)+B K(x, x)\left(\begin{array}{c}
\sin \lambda x \\
-\cos \lambda x
\end{array}\right)
$$




$$
+\int_{0}^{x} B K_{x}^{\prime}(x, t)\left(\begin{array}{c}
\sin \lambda t \\
-\cos \lambda t
\end{array}\right) d t .
$$

On the other hand, multiplying (2.2) on the left by $\lambda$ and integrating by parts and using (5.6), we find

$$
\begin{aligned}
\lambda \varphi(x, \lambda)= & \lambda B\left(\begin{array}{c}
\cos \lambda x \\
\sin \lambda x
\end{array}\right)+K(x, x) B\left(\begin{array}{c}
\sin \lambda x \\
-\cos \lambda x
\end{array}\right) \\
& -\int_{0}^{x} K_{t}^{\prime}(x, t) B\left(\begin{array}{c}
\sin \lambda t \\
-\cos \lambda t
\end{array}\right) d t .
\end{aligned}
$$

It follows from (5.13) and (5.14) that

$$
\begin{aligned}
\lambda \varphi(x, \lambda)= & B \varphi_{x}^{\prime}(x, \lambda)+[K(x, x) B-B K(x, x)]\left(\begin{array}{c}
\sin \lambda x \\
-\cos \lambda x
\end{array}\right) \\
& -\int_{0}^{x}\left[K_{t}^{\prime}(x, t) B+B K_{x}^{\prime}(x, t)\right]\left(\begin{array}{c}
\sin \lambda t \\
-\cos \lambda t
\end{array}\right) d t .
\end{aligned}
$$

Taking into account (2.3) and (5.12),

$$
B \varphi_{x}^{\prime}(x, \lambda)+\Omega(x) \varphi(x, \lambda)=\lambda \varphi(x, \lambda)
$$

is obtained. For $x=0$, from (2.2) we get (5.2).

\subsection{Derivation of Parseval equality}

Theorem 6. For any $g(x) \in L_{2}\left(0, \pi ; \mathbb{C}^{2}\right)$, the following relation holds:

$$
\|g\|_{L_{2}}^{2}=\sum_{n=-\infty}^{\infty} \frac{1}{\alpha_{n}}\left(\int_{0}^{\pi} \tilde{\varphi}\left(t, \lambda_{n}\right) g(t) d t\right)^{2} .
$$

Proof. Denote

It follows from (2.2) that

$$
Q(\lambda):=\int_{0}^{\pi} \tilde{\varphi}(t, \lambda) g(t) d t .
$$

$$
Q(\lambda):=\int_{0}^{\pi} \tilde{\varphi}_{0}(t, \lambda) h(t) d t
$$

where

$$
h(t)=g(t)+\int_{t}^{\pi} \tilde{K}(s, t) g(s) d s .
$$

Similarly, in view of (3.3),

$$
g(t)=h(t)+\int_{t}^{\pi} \tilde{H}(s, t) h(s) d s
$$

where for the kernel $H(x, t)$ we have the identity

$$
\tilde{H}(x, t)=F(t, x)+\int_{0}^{t} K(t, \xi) F(\xi, x) d \xi .
$$


Using (5.16), we get

$$
\begin{aligned}
\int_{0}^{\pi} F(x, t) h(t) d t & =\int_{0}^{\pi} F(x, t)\left[g(t)+\int_{t}^{\pi} \tilde{K}(s, t) g(s) d s\right] d t \\
& =\int_{0}^{\pi}\left[F(x, t)+\int_{0}^{t} F(x, s) \tilde{K}(t, s) d s\right] g(t) d t \\
& =\int_{0}^{x}\left[F(x, t)+\int_{0}^{t} F(x, s) \tilde{K}(t, s) d s\right] g(t) d t \\
& +\int_{x}^{\pi}\left[F(x, t)+\int_{0}^{t} F(x, s) \tilde{K}(t, s) d s\right] g(t) d t .
\end{aligned}
$$

It follows from (3.1) and (5.18) that

$$
\int_{0}^{\pi} F(x, t) h(t) d t=\int_{0}^{x} H(x, t) g(t) d t-\int_{x}^{\pi} \tilde{K}(t, x) g(t) d t .
$$

From (3.2) and Parseval equality, we obtain

$$
\begin{aligned}
& \int_{0}^{\pi}\left[h_{1}^{2}(t)+h_{2}^{2}(t)\right] d t+\int_{0}^{\pi} \int_{0}^{\pi} \tilde{h}(x) F(x, t) h(t) d t d x \\
& =\int_{0}^{\pi}\left[h_{1}^{2}(t)+h_{2}^{2}(t)\right] d t+\sum_{n=-\infty}^{\infty} \frac{1}{\alpha_{n}}\left(\int_{0}^{\pi} \tilde{\varphi}_{0}\left(t, \lambda_{n}\right) h(t) d t\right)^{2} \\
& \quad-\sum_{n=-\infty}^{\infty} \frac{1}{\pi}\left(\int_{0}^{\pi} \tilde{\varphi}_{0}\left(t, \lambda_{n}^{0}\right) h(t) d t\right)^{2} \\
& =\sum_{n=-\infty}^{\infty} \frac{1}{\alpha_{n}}\left(\int_{0}^{\pi} \tilde{\varphi}_{0}\left(t, \lambda_{n}\right) h(t) d t\right)^{2} \\
& =\sum_{n=-\infty}^{\infty} \frac{Q^{2}\left(\lambda_{n}\right)}{\alpha_{n}} .
\end{aligned}
$$

Taking into account (5.19), we have

$$
\begin{aligned}
\sum_{n=-\infty}^{\infty} \frac{Q^{2}\left(\lambda_{n}\right)}{\alpha_{n}}= & \int_{0}^{\pi}\left[h_{1}^{2}(t)+h_{2}^{2}(t)\right] d t+\int_{0}^{\pi} \tilde{h}(x)\left(\int_{0}^{x} H(x, t) g(t) d t\right) d x \\
& -\int_{0}^{\pi} \tilde{h}(x)\left(\int_{x}^{\pi} \tilde{K}(t, x) g(t) d t\right) d x \\
= & \int_{0}^{\pi}\left[h_{1}^{2}(t)+h_{2}^{2}(t)\right] d t+\int_{0}^{\pi}\left(\int_{t}^{\pi} \tilde{h}(x) H(x, t) d x\right) g(t) d t \\
& -\int_{0}^{\pi} \tilde{h}(x)\left(\int_{x}^{\pi} \tilde{K}(t, x) g(t) d t\right) d x,
\end{aligned}
$$


whence by formulas (5.16) and (5.17),

$$
\begin{aligned}
\sum_{n=-\infty}^{\infty} \frac{Q^{2}\left(\lambda_{n}\right)}{\alpha_{n}}= & \int_{0}^{\pi}\left[h_{1}^{2}(t)+h_{2}^{2}(t)\right] d t+\int_{0}^{\pi}(\tilde{g}(t)-\tilde{h}(t)) g(t) d t \\
& -\int_{0}^{\pi} \tilde{h}(x)(h(x)-g(x)) d x \\
= & \int_{0}^{\pi}\left(g_{1}^{2}(t)+g_{2}^{2}(t)\right) d t
\end{aligned}
$$

is obtained, i.e., the relation (5.15) is valid.

Corollary 1. For any function $f(x)$ and $g(x) \in L_{2}\left(0, \pi ; \mathbb{C}^{2}\right)$, the following relation holds:

$$
\int_{0}^{\pi} \tilde{g}(x) f(x) d x=\sum_{n=-\infty}^{\infty} \frac{1}{\alpha_{n}}\left(\int_{0}^{\pi} \tilde{g}(t) \varphi\left(t, \lambda_{n}\right) d t\right)\left(\int_{0}^{\pi} \tilde{\varphi}\left(t, \lambda_{n}\right) f(t) d t\right) .
$$

Lemma 2. For any $f(x) \in W_{2}^{1}[0, \pi]$, the expansion formula

$$
f(x)=\sum_{n=-\infty}^{\infty} c_{n} \varphi\left(x, \lambda_{n}\right)
$$

is valid, where

$$
c_{n}=\frac{1}{\alpha_{n}} \int_{0}^{\pi} \tilde{\varphi}\left(x, \lambda_{n}\right) f(x) d x
$$

Proof. Consider the series

$$
f^{*}(x)=\sum_{n=-\infty}^{\infty} c_{n} \varphi\left(x, \lambda_{n}\right),
$$

where

$$
c_{n}:=\frac{1}{\alpha_{n}} \int_{0}^{\pi} \tilde{\varphi}\left(x, \lambda_{n}\right) f(x) d x .
$$

Using Theorem 2.5 and integrating by parts, we get

$$
\begin{aligned}
c_{n}= & \frac{1}{\alpha_{n} \lambda_{n}} \int_{0}^{\pi}\left[-\frac{\partial}{\partial x} \tilde{\varphi}\left(x, \lambda_{n}\right) B+\tilde{\varphi}\left(x, \lambda_{n}\right) \Omega(x)\right] f(x) d x \\
= & -\frac{1}{\alpha_{n} \lambda_{n}}\left[\tilde{\varphi}\left(\pi, \lambda_{n}\right) B f(\pi)-\tilde{\varphi}\left(0, \lambda_{n}\right) B f(0)\right] \\
& +\frac{1}{\alpha_{n} \lambda_{n}} \int_{0}^{\pi} \tilde{\varphi}\left(x, \lambda_{n}\right)\left[B f^{\prime}(x)+\Omega(x) f(x)\right] d x .
\end{aligned}
$$


Applying the asymptotic formulas in Theorem 1.1, we find $\left\{c_{n}\right\} \in l_{2}$. Consequently the series (5.22) converges absolutely and uniformly on $[0, \pi]$. According to $(5.20)$ and (5.23), we have

$$
\begin{aligned}
\int_{0}^{\pi} \tilde{g}(x) f(x) d x & =\sum_{n=-\infty}^{\infty} \frac{1}{\alpha_{n}}\left(\int_{0}^{\pi} \tilde{g}(t) \varphi\left(t, \lambda_{n}\right) d t\right)\left(\int_{0}^{\pi} \tilde{\varphi}\left(t, \lambda_{n}\right) f(t) d t\right) \\
& =\sum_{n=-\infty}^{\infty} c_{n}\left(\int_{0}^{\pi} \tilde{g}(t) \varphi\left(t, \lambda_{n}\right) d t\right)=\int_{0}^{\pi} \tilde{g}(t)\left(\sum_{n=-\infty}^{\infty} c_{n} \varphi\left(t, \lambda_{n}\right)\right) \\
& =\int_{0}^{\pi} \tilde{g}(t) f^{*}(t) d t .
\end{aligned}
$$

Since $g(x)$ is arbitrary, $f(x)=f^{*}(x)$ is obtained, i.e., the expansion formula (5.21) is proved.

\subsection{Derivation of boundary condition}

Lemma 3. The following equality holds:

$$
\sum_{n=-\infty}^{\infty} \frac{\varphi\left(x, \lambda_{n}\right)}{\alpha_{n} \beta_{n}}=0
$$

Proof. Using residue theorem, we get

$$
\sum_{n=-\infty}^{\infty} \frac{\varphi\left(x, \lambda_{n}\right)}{\alpha_{n} \beta_{n}}=\sum_{n=-\infty}^{\infty} \frac{\varphi\left(x, \lambda_{n}\right)}{\dot{\Delta}\left(\lambda_{n}\right)}=\sum_{n=-\infty}^{\infty} \underset{\lambda=\lambda_{n}}{\operatorname{Res}} \frac{\varphi(x, \lambda)}{\Delta(\lambda)}=\frac{1}{2 \pi i} \int_{\Gamma_{N}} \frac{\varphi(x, \lambda)}{\Delta(\lambda)} d \lambda,
$$

where $\Gamma_{N}=\left\{\lambda:|\lambda|=N+\frac{1}{2}\right\}$. From (2.12) and ([19], Lemma 3.4.2),

$$
\Delta(\lambda)=\lambda \sin \lambda \pi+O\left(e^{|\operatorname{Im} \lambda| \pi}\right) .
$$

We denote $G_{\delta}=\{\lambda:|\lambda-n| \geq \delta, n=0, \pm 1, \pm 2 \ldots\}$ for some small fixed $\delta>0$ and $|\sin \lambda \pi| \geq C_{\delta} e^{|\operatorname{Im} \lambda| \pi}, \lambda \in G_{\delta}$, where $C_{\delta}$ positive number. Therefore, we have

$$
|\Delta(\lambda)| \geq C_{\delta}|\lambda| e^{|I m \lambda| \pi}, \quad \lambda \in G_{\delta} .
$$

Using this inequality and (2.10), we obtain (5.24).

Theorem 7. The following relation is valid:

$$
\left(\lambda_{n}+h_{1}\right) \varphi_{1}\left(\pi, \lambda_{n}\right)+h_{2} \varphi_{2}\left(\pi, \lambda_{n}\right)=0 .
$$

Proof. From (5.24), we can write for any $n_{0} \in \mathbb{Z}$

$$
\frac{\varphi\left(x, \lambda_{n_{0}}\right)}{\alpha_{n_{0}}}=-\sum_{\substack{n=-\infty \\ n \neq n_{0}}}^{\infty} \frac{\beta_{n_{0}} \varphi\left(x, \lambda_{n}\right)}{\alpha_{n} \beta_{n}}
$$


Let $k \neq n_{0}$ be any fixed number and $f(x)=\varphi\left(x, \lambda_{k}\right)$. Then substituting (5.28) in (5.21)

$$
\varphi\left(x, \lambda_{k}\right)=\sum_{\substack{n=-\infty \\ n \neq n_{0}}}^{\infty} c_{n k} \varphi\left(x, \lambda_{n}\right)
$$

where

$$
c_{n k}=\frac{1}{\alpha_{n}} \int_{0}^{\pi}\left[\tilde{\varphi}\left(t, \lambda_{n}\right)-\frac{\beta_{n_{0}}}{\beta_{n}} \tilde{\varphi}\left(t, \lambda_{n_{0}}\right)\right] \varphi\left(t, \lambda_{k}\right) d t .
$$

The system of functions $\left\{\varphi_{0}\left(x, \lambda_{n}\right)\right\},(n \in \mathbb{Z})$ is orthogonal in $L_{2}\left(0, \pi ; \mathbb{C}^{2}\right)$. Then by (2.2), the system of functions $\left\{\varphi\left(x, \lambda_{n}\right)\right\},(n \in \mathbb{Z})$ is orthogonal in $L_{2}\left(0, \pi ; \mathbb{C}^{2}\right)$ as well. Therefore, $c_{n k}=\delta_{n k}$, where $\delta_{n k}$ is Kronecker delta. Let us define

$$
a_{n k}:=\int_{0}^{\pi} \tilde{\varphi}\left(t, \lambda_{n}\right) \varphi\left(t, \lambda_{k}\right) d t
$$

Using this expression, we have for $n \neq k$

$$
a_{k k}-\frac{\beta_{n}}{\beta_{k}} a_{n k}=\alpha_{k}
$$

It follows from (5.29) that $a_{n k}=a_{k n}$. Taking into account this equality and (5.30),

$$
\beta_{k}^{2}\left(\alpha_{k}-a_{k k}\right)=\beta_{n}^{2}\left(\alpha_{n}-a_{n n}\right)=H, \quad k \neq n,
$$

where $H$ is a constant. Then, we have

$$
\int_{0}^{\pi} \tilde{\varphi}\left(t, \lambda_{n}\right) \varphi\left(t, \lambda_{n}\right) d t=\alpha_{n}-\frac{H}{\beta_{n}^{2}}
$$

and

$$
\int_{0}^{\pi} \tilde{\varphi}\left(t, \lambda_{k}\right) \varphi\left(t, \lambda_{n}\right) d t=-\frac{H}{\beta_{k} \beta_{n}}, k \neq n .
$$

It is easily obtained that for $k \neq n$,

$$
\begin{aligned}
\int_{0}^{\pi}\left[\varphi_{1}\left(x, \lambda_{k}\right) \varphi_{1}\left(x, \lambda_{n}\right)+\varphi_{2}\left(x, \lambda_{k}\right) \varphi_{2}\left(x, \lambda_{n}\right)\right] d x \\
\quad=\frac{1}{\left(\lambda_{k}-\lambda_{n}\right)}\left[\varphi_{2}\left(\pi, \lambda_{k}\right) \varphi_{1}\left(\pi, \lambda_{n}\right)-\varphi_{1}\left(\pi, \lambda_{k}\right) \varphi_{2}\left(\pi, \lambda_{n}\right)\right]=-\frac{H}{\beta_{k} \beta_{n}} .
\end{aligned}
$$

According to the last equation, for $n \neq k$,

$$
\beta_{k} \varphi_{2}\left(\pi, \lambda_{k}\right) \beta_{n} \varphi_{1}\left(\pi, \lambda_{n}\right)-\beta_{k} \varphi_{1}\left(\pi, \lambda_{k}\right) \beta_{n} \varphi_{2}\left(\pi, \lambda_{n}\right)=-H\left(\lambda_{k}-\lambda_{n}\right) .
$$

We denote

$$
D_{n}:=\beta_{n} \varphi_{1}\left(\pi, \lambda_{n}\right), \quad E_{n}:=\beta_{n} \varphi_{2}\left(\pi, \lambda_{n}\right) .
$$

Then, we can rewrite equation (5.31) as follows

$$
D_{k} E_{n}-E_{k} D_{n}=H\left(\lambda_{k}-\lambda_{n}\right), \quad n \neq k .
$$


Let $i, j, k, n$ be pairwise distinct integers, then we get

$$
\begin{aligned}
D_{k} E_{n}-E_{k} D_{n} & =H\left(\lambda_{k}-\lambda_{n}\right), \\
D_{n} E_{i}-E_{n} D_{i} & =H\left(\lambda_{n}-\lambda_{i}\right), \\
D_{i} E_{k}-E_{i} D_{k} & =H\left(\lambda_{i}-\lambda_{k}\right) .
\end{aligned}
$$

Adding them together, we find

$$
D_{n}\left(E_{i}-E_{k}\right)+E_{n}\left(D_{k}-D_{i}\right)=E_{i} D_{k}-D_{i} E_{k} .
$$

In this equation, replacing $n$ by $j$, we get another equation

$$
D_{j}\left(E_{i}-E_{k}\right)+E_{j}\left(D_{k}-D_{i}\right)=E_{i} D_{k}-D_{i} E_{k} .
$$

Subtracting the last two equation,

$$
\left(D_{n}-D_{j}\right)\left(E_{i}-E_{k}\right)=\left(D_{i}-D_{k}\right)\left(E_{n}-E_{j}\right) .
$$

In the case of $E_{n}=E_{j}$, for some $n, j \in \mathbb{Z}$, then $E_{n}=$ const. From (5.33), $D_{n}=$ $\mu_{1} \lambda_{n}+\mu_{2}$. In the case of $E_{n} \neq E_{j}$, then we obtain $D_{n}=\mu_{1} \lambda_{n}+\mu_{2}$ and $E_{n}=$ $\mu_{3} \lambda_{n}+\mu_{4}$, where in both cases $\mu_{1}, \mu_{2}, \mu_{3}, \mu_{4}$ are constant. Therefore, using these relation in (5.32), we find

$$
\beta_{n} \varphi_{1}\left(\pi, \lambda_{n}\right)=\mu_{1} \lambda_{n}+\mu_{2}, \quad \beta_{n} \varphi_{2}\left(\pi, \lambda_{n}\right)=\mu_{3} \lambda_{n}+\mu_{4} .
$$

Using

$$
\varphi_{1}\left(\pi, \lambda_{n}\right)=O\left(\frac{1}{n}\right), \quad \varphi_{2}\left(\pi, \lambda_{n}\right)=(-1)^{n+1}+O\left(\frac{1}{n}\right),
$$

$\lambda_{n}=n+O\left(\frac{1}{n}\right)$ and $\beta_{n}=n(-1)^{n}+O(1)$ derived from (2.6) and (5.26), we obtain $\mu_{1}=0, \mu_{3}=-1$. Denoting $h_{2}:=\mu_{2}$ and $h_{1}:=-\mu_{4}$,

$$
h_{2} \varphi_{2}\left(\pi, \lambda_{n}\right)=-\left(\lambda_{n}+h_{1}\right) \varphi_{1}\left(\pi, \lambda_{n}\right), n \in \mathbb{Z}
$$

is obtained and it follows from (5.33) that $H=h_{2}$.

Thus, we have proved the following theorem:

Theorem 8. For the sequences $\left\{\lambda_{n}, \alpha_{n}\right\},(n \in \mathbb{Z})$, to be the spectral data for a certain boundary value problem $L\left(\Omega(x), h_{1}, h_{2}\right)$ of the form (1.1), (1.2) with $\Omega(x) \in$ $L_{2}(0, \pi)$ it is necessary and sufficient that the relations (2.7) and (2.9) hold.

Algorithm for construction of the function $\Omega(x)$ by spectral data $\left\{\lambda_{n}, \alpha_{n}\right\},(n \in \mathbb{Z})$ follows from the proof of the theorem:

1) By the given numbers $\left\{\lambda_{n}, \alpha_{n}\right\},(n \in \mathbb{Z})$ the function $F(x, t)$ is constructed by formula (3.2),

2) The function $K(x, t)$ is found from equation (3.1),

3) $\Omega(x)$ is calculated by the formula (2.3). 


\section{ACKNOWLEDGEMENT}

This work is supported by The Scientific and Technological Research Council of Turkey (TÜBİTAK).

\section{REFERENCES}

[1] M. J. Ablowitz, D. J. Kaup, A. C. Newell, and H. Segur, "Nonlinear-evolution equations of physical significance," Phys. Rev. Lett., vol. 31, pp. 125-127, 1973.

[2] S. Albeverio, R. Hryniv, and Y. Mykytyuk, "Inverse spectral problems for Dirac operators with summable potentials,” Russ. J. Math. Phys., vol. 12, pp. 406-423, 2005.

[3] R. K. Amirov, B. Keskin, and A. S. Ozkan, "Direct and inverse problems for the Dirac operator with spectral parameter linearly contained in boundary condition," Ukrainian Math. J., vol. 61, no. 9, pp. 1365-1379, 2009.

[4] A. Çöl and K. R. Mamedov, "On an inverse scattering problem for a class of Dirac operators with spectral parameter in the boundary condition," J. Math. Anal. Appl., vol. 393, pp. 470-478, 2012.

[5] A. B. de Monvel and D. Shepelsky, "Initial boundary value problem for the $\mathrm{m} K \mathrm{~d} V$ equation on a finite interval," Ann. Inst. Fourier, Grenoble, vol. 54, no. 5, pp. 1477-1495, 2004.

[6] T. Dzabiev, "The inverse problem for the Dirac equation with a singularity," Dokl. Akad. Nauk Azerbaidzan SSR, vol. 22, no. 11, pp. 8-11, 1966.

[7] G. Freiling and V. Yurko, Inverse Sturm-Liouville Problems and Their Applications. Huntington, New York: Nova Science Publisher, 2008.

[8] B. Fritzsche, B. Kirstein, I. Y. Roitberg, and A. Sakhnovich, "Skew-self-adjoint Dirac system with a rectangular matrix potential: $W$ eyl theory, direct and inverse problems," Integral Equations and Operator Theory, vol. 74, no. 2, pp. 163-187, 2012.

[9] M. G. Gasymov, "The inverse scattering problem for a system of Dirac equations of order $2 n$," Trans. Moskow Math. Soc., vol. 19, pp. 41-119, 1968.

[10] M. G. Gasymov and T. Dzabiev, "Solution of the inverse problem by two spectra for the Dirac equation on a finite interval," Dokl. Akad. Nauk Azerbaidzan SSR, vol. 22, no. 7, pp. 3-6, 1966.

[11] M. Horvath, "On the inverse spectral theory of $S$ chrödinger and Dirac operators," Trans. Amer. Math. Soc., vol. 353, pp. 4155-4171, 2001.

[12] M. G. Krein, "On integral equations generating differential equations of the second order," Dokl. Akad. SSSR., vol. 97, pp. 21-24, 1954.

[13] Y. B. Levin, Lectures on Entire Functions. Providence: American Mathematical Society, 1996.

[14] B. M. Levitan and I. S. Sargsjan, Strum-Liouville and Dirac Operator. Dordrecht, Boston, London: Kluwer Academic Publisher, 1991.

[15] K. R. Mamedov and O. Akcay, "Necessary and sufficient conditions for the solvability of inverse problem for a class of Dirac operators," arXiv:1404.0192, 2014.

[16] K. R. Mamedov and O. Akçay, "Inverse problem for a class of Dirac operator," Taiwanese Journal of Mathematics, vol. 18, no. 3, pp. 753-772, 2014.

[17] K. R. Mamedov and A. Çöl, "On an inverse scattering problem for a class Dirac operator with discontinuous coefficient and nonlinear dependence on the spectral parameter in the boundary condition," Mathematical Methods in the Applied Sciences, vol. 35, no. 14, pp. 1712-1720, 2012.

[18] S. G. Mamedov, "The inverse boundary value problem on a finite interval for Dirac's system of equations," Azerbaidzan Gos. Univ. Ucen. Zap. Ser. Fiz-Mat. Nauk, no. 5, pp. 61-67, 1975.

[19] V. A. Marchenko, Sturm-Liouville Operators and Applications, 2nd ed. Providence, Rhode Island,: AMS Chelsea Publishing, 2011.

[20] K. Mochizuki and I. Trooshin, "Inverse problem for interior spectral data of the Dirac operator on a finite interval," Publ. RIMS, Kyoto Univ., vol. 38, pp. 387-395, 2002. 
[21] Y. Mykytyuk and V. Puyda, "Inverse spectral problems for Dirac operators on a finite interval," $J$. Math. Anal. Appl., vol. 386, no. 1, pp. 177-194, 2012.

[22] A. Sakhnovich, "Skew-self-adjoint discrete and continuous Dirac-type systems: inverse problems and Borg-M archenko theorems," Inverse Problems, vol. 22, no. 6, pp. 2083-2101, 2006.

[23] D. G. Shepelsky, "An inverse spectral problem for a Dirac-type operator with sewing," in Dynamical Systems and Complex Analysis. Kiev: Naukova Dumka, 1992, pp. 104-112.

[24] B. Thaller, The Dirac Equation. Berlin: Springer, 1992.

[25] B. A. Watson, "Inverse spectral problems for weighted Dirac systems," Inverse Problems, vol. 15, pp. 793-805, 1999.

Authors' addresses

Kh. R. Mamedov

Mersin University, Mathematics Department, 33343 Mersin, Turkey,

Institute of Mathematics and Mechanics of NAS of Azerbaijan, AZ1141 Baku, Azerbaijan

E-mail address: hanlar@mersin.edu.tr

O. Akcay

Mersin University, Mathematics Department, 33343 Mersin, Turkey

E-mail address: ozge.akcy@gmail.com 